\title{
ON THE REGULARIZING PROPERTIES OF THE GMRES METHOD
}

\author{
D. CALVETTI *, B. LEWIS $\dagger$, AND L. REICHEL ‡
}

\begin{abstract}
The GMRES method is a popular iterative method for the solution of large linear systems of equations with a nonsymmetric nonsingular matrix. However, little is known about the behavior of this method when it is applied to the solution of nonsymmetric linear ill-posed problems with a right-hand side that is contaminated by errors. We show that when the associated error-free right-hand side lies in a finite-dimensional Krylov subspace, the GMRES method is a regularization method. The iterations are terminated by a stopping rule based on the discrepancy principle.
\end{abstract}

Key words. Regularization, ill-posed problems, GMRES.

AMS subject classifications. $65 \mathrm{~F} 10,65 \mathrm{~F} 22,65 \mathrm{R} 30$

1. Introduction. Let $\mathcal{X}$ be a real separable Hilbert space with inner product $<\cdot, \cdot>$ and norm $\|\cdot\|=<\cdot, \cdot\rangle^{1 / 2}$, and let $A: \mathcal{X} \rightarrow \mathcal{X}$ be a bounded linear operator. The induced operator norm is also denoted by $\|\cdot\|$. Let $\mathcal{R}(A)$ denote the range of $A$ and assume that for every $b \in \mathcal{R}(A)$ the equation

$$
A x=b
$$

has a unique solution $x \in \mathcal{X}$. Thus, the operator $A$ has an inverse on $\mathcal{R}(A)$, which we denote by $A^{-1}$. We are interested in the situation when the solution $x$ of (1.1) does not depend continuously on the the right-hand side $b$, i.e., when the inverse operator $A^{-1}$ is not bounded on $\mathcal{R}(A)$. Then the determination of the solution $x$ of (1.1) is an ill-posed problem in the sense of Hadamard, and we refer to equation (1.1) as an ill-posed problem; see, e.g., Groetsch [7, Chapter 1] for a discussion on ill-posed problems.

In many linear ill-posed problems (1.1) that arise in science and engineering, the right-hand side $b$, which is assumed to be in $\mathcal{R}(A)$, is not available. Instead, a perturbation $b^{\delta} \in \mathcal{X}$ of $b$ is known. The difference $b-b^{\delta}$ often stems from measurement errors and is referred to as "noise." In the present paper, we assume that a bound $\delta \geq 0$ of the norm of the noise is known, i.e.,

$$
\left\|b-b^{\delta}\right\| \leq \delta .
$$

We would like to compute an approximate solution of equation (1.1) with the unknown right-hand side $b$ by computing an approximate solution of the equation

$$
A x=b^{\delta} .
$$

Two possible difficulties may arise. The perturbed right-hand side $b^{\delta}$ might be in $\mathcal{X} \backslash \mathcal{R}(A)$, and then equation (1.3) does not have a solution. Further, when $b^{\delta} \in \mathcal{R}(A)$, the solution $x^{\delta}$ of (1.3) may be a poor approximation of the solution $x$ of (1.1) even when $b$ and $b^{\delta}$ are close, because $A^{-1}$ is not bounded. Therefore, the determination of a solution of (1.3), if it exists, is an ill-posed problem.

\footnotetext{
* Department of Mathematics, Case Western Reserve University, Cleveland, OH 44106. E-mail: dxc57@po.cwru.edu. Research supported in part by NSF grant DMS-9806702.

${ }^{\dagger}$ Department of Mathematics and Computer Science, Kent State University, Kent, OH 44242. E-mail: blewis@mcs.kent.edu. Research supported in part by NSF grant DMS-9806413.

${ }^{\ddagger}$ Department of Mathematics and Computer Science, Kent State University, Kent, OH 44242. E-mail: reichel@mcs.kent.edu. Research supported in part by NSF grant DMS-9806413.
} 
Due to these difficulties, we do not try to compute the solution $x^{\delta}$ of (1.3), even if it exists. Instead, for a given right-hand side $b^{\delta} \in \mathcal{X}$ that satisfies (1.2), we replace the operator $A$ in (1.3) by an operator $A_{\text {reg }}^{\delta}: \mathcal{X} \rightarrow \mathcal{X}$ that approximates $A$ and has a bounded inverse on $\mathcal{X}$, and solve the equation

$$
A_{r e g}^{\delta} x=b^{\delta} .
$$

The replacement of the operator $A$ by $A_{\text {reg }}^{\delta}$ is referred to as regularization, $A_{\text {reg }}^{\delta}$ as a regularized operator and the solution $x_{r e g}^{\delta}$ of (1.4) as a regularized approximate solution of (1.3). We would like to choose $A_{r e g}^{\delta}$ so that $x_{r e g}^{\delta}$ is a meaningful approximation of the solution of the equation (1.1) with unknown right-hand side. When a bound of the norm of the noise $\delta$ in the right-hand side $b^{\delta}$ is known, the operator $A_{\text {reg }}^{\delta}$ is commonly chosen so that the norm of the discrepancy

$$
d_{\text {reg }}^{\delta}=b^{\delta}-A x_{r e g}^{\delta}
$$

associated with $x_{r e g}^{\delta}$ is of the order of magnitude $\delta$. Then $x_{r e g}^{\delta}$ satisfies the discrepancy principle defined as follows.

Definition (Discrepancy Principle). Let $\alpha>0$ be fixed and let $b^{\delta} \in \mathcal{X}$ satisfy (1.2) for some $\delta \geq 0$. The regularized approximate solution $x_{r e g}^{\delta}$ of (1.3) is said to satisfy the discrepancy principle if $\left\|b^{\delta}-A x_{r e g}^{\delta}\right\| \leq \alpha \delta$.

In the development below, we will keep $\alpha>0$ fixed and investigate the convergence of $x_{r e g}^{\delta}$ to the solution of (1.1) as $\delta$ converges to zero.

One of the most popular regularization methods is Tikhonov regularization, which in its simplest form yields an operator $A_{\text {reg }}^{\delta}$ with inverse

$$
\left(A_{\text {reg }}^{\delta}\right)^{-1}=\left(A^{*} A+\mu I\right)^{-1} A^{*} ;
$$

see Groetsch [7] for a thorough discussion. Here $A^{*}$ denotes the adjoint operator to $A$ and $I$ denotes the identity operator. The parameter $\mu \geq 0$ is referred to as a regularization parameter. It determines how sensitive the solution $x_{r e g}^{\delta}$ of (1.4) is to perturbations in the right-hand side $b^{\delta}$ and how close $x_{r e g}^{\delta}$ is to the solution $x$ of (1.1).

In the present paper, we define the operator $A_{\text {reg }}^{\delta}$ by applying a few steps of the Generalized Minimal Residual (GMRES) iterative method, due to Saad and Schultz [16], to equation (1.3). The GMRES method is a popular iterative method for the solution of equations of the form (1.1) with a bounded operator $A$ with a bounded inverse. It is the purpose of this paper to investigate the behavior of the GMRES method when it is applied to the computation of approximate solutions of equations of the form (1.3) with a bounded nonsymmetric operator with an unbounded inverse and a right-hand side that is contaminated by noise. We show that under appropriate conditions on the unperturbed equation (1.1), the GMRES method equipped with a stopping rule based on the Discrepancy Principle is a regularization method.

Related investigations for linear ill-posed problems (1.3) with a symmetric operator $A$ have been presented by Hanke [8]. In particular, Hanke [8, Chapters 3 and 6] shows that the conjugate residual method and a variant thereof, the MR-II method, which are minimal residual methods for equations with a symmetric operator, are regularization methods when equipped with a stopping rule based on the Discrepancy Principle. Studies of the behavior of the conjugate gradient method applied to the normal equations associated with (1.3),

$$
A^{*} A x=A^{*} b^{\delta}
$$


can be found in $[8,9,11]$. Plato [14] studied simple iterative methods, such as Richardson iteration, for the solution of linear ill-posed problems (1.3) with a nonsymmetric operator. We are not aware of investigations of more general Krylov methods for the solution of linear ill-posed problems with a nonsymmetric operator.

When the regularized operator $A_{\text {reg }}^{\delta}$ is defined by taking $m$ steps of the GMRES method, the number of steps can be thought of as a regularization parameter. In order to emphasize the dependence on $m$, we denote the regularized operator defined by taking $m$ steps of the GMRES method by $A_{m}^{\delta}$, its inverse by $\left(A_{m}^{\delta}\right)^{-1}$ and the computed solution of (1.4) with initial approximate solution $x_{0}^{\delta}=0$ by $x_{m}^{\delta}$, i.e.,

$$
x_{m}^{\delta}=\left(A_{m}^{\delta}\right)^{-1} b^{\delta} .
$$

We remark that the bounded linear operator $\left(A_{m}^{\delta}\right)^{-1}$ depends not only on the number of steps $m$ of the GMRES method and on the initial approximate solution $x_{0}^{\delta}$, but also on the right-hand side $b^{\delta}$. Throughout this paper, we will choose the initial approximate solution $x_{0}^{\delta}=0$.

In general, the iterates $x_{m}^{\delta}$ do not converge to the solution $x$ of (1.1) as the iteration number $m$ increases; in fact, $\left\|x-x_{m}^{\delta}\right\|$ typically grows with $m$, for $m$ sufficiently large. It is therefore important to terminate the iterations at a suitable step. The following stopping rule terminates the iterations as soon as an iterate $x_{m}^{\delta}$ that satisfies the discrepancy principle has been found.

Stopping Rule 1.1. Let $\alpha$ and $\delta$ be the same as in the Discrepancy Principle. Denote the iterates determined by the GMRES method applied to the solution of (1.3) by $x_{m}^{\delta}, m=1,2, \ldots$. Terminate the iterations as soon as an iterate has been determined, such that

$$
\left\|b^{\delta}-A x_{m}^{\delta}\right\| \leq \alpha \delta
$$

We denote the termination index by $m_{\delta}$.

We say that an iterative method for (1.3) equipped with this stopping rule is a regularization method if there is a constant $\alpha>0$, independent of $\delta$, such that the iterates $x_{m_{\delta}}^{\delta}$ defined by (1.7), with $m=m_{\delta}$ determined by the stopping rule, satisfy

$$
\lim _{\delta \searrow 0} \sup _{\left\|b-b^{\delta}\right\| \leq \delta}\left\|x-x_{m_{\delta}}^{\delta}\right\|=0
$$

where $x$ solves (1.1).

This paper is organized as follows. Basic properties of the GMRES method are reviewed in Section 2. Regularizing properties are studied in Section 3, and a few computed examples that illustrate the behavior of the limit (1.8) are presented in Section 4 .

2. The GMRES method. Introduce the Krylov subspaces

$$
\mathcal{K}_{m}\left(A, b^{\delta}\right)=\operatorname{span}\left\{b^{\delta}, A b^{\delta}, A^{2} b^{\delta}, \ldots, A^{m-1} b^{\delta}\right\}, \quad m=1,2, \ldots .
$$

The GMRES method by Saad and Schultz [16] applied to the solution of equation (1.3) with initial approximate solution $x_{0}^{\delta}=0$ determines iterates $x_{m}^{\delta}$ that satisfy

$$
\left\|A x_{m}^{\delta}-b^{\delta}\right\|=\min _{x \in \mathcal{K}_{m}\left(A, b^{\delta}\right)}\left\|A x-b^{\delta}\right\|, \quad x_{m}^{\delta} \in \mathcal{K}_{m}\left(A, b^{\delta}\right), \quad m=1,2, \ldots
$$


Saad and Schultz [16] propose to compute the iterate $x_{m}^{\delta}$ by first determining an orthonormal basis $\left\{v_{j}^{\delta}\right\}_{j=1}^{m}$ of the Krylov subspace $\mathcal{K}_{m}\left(A, b^{\delta}\right)$ by the Arnoldi process. In the description of the algorithm below, we tacitly assume that

$$
\operatorname{dim} \mathcal{K}_{m}\left(A, b^{\delta}\right)=m .
$$

The condition (2.3) secures that the Arnoldi process does not break down before an orthonormal basis $\left\{v_{j}^{\delta}\right\}_{j=1}^{m}$ of $\mathcal{K}_{m}\left(A, b^{\delta}\right)$ with $m$ elements has been determined. We will return to this assumption below.

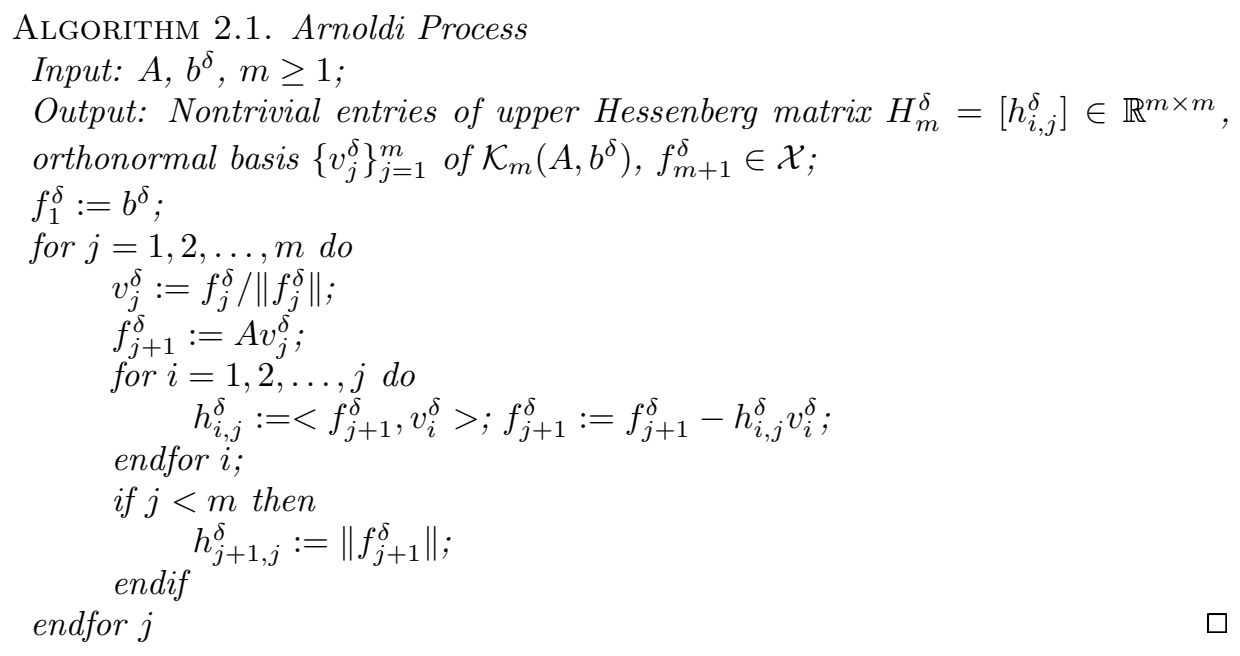

Let $e_{j}=[0, \ldots, 0,1,0, \ldots, 0]^{T}$ denote the $j$ th axis vector of $\mathbb{R}^{m}$, and define the operator $V_{m}^{\delta}: \mathbb{R}^{m} \rightarrow \mathcal{K}_{m}\left(A, b^{\delta}\right)$ by

$$
V_{m}^{\delta} y=\sum_{j=1}^{m}\left(e_{j}^{T} y\right) v_{j}^{\delta}, \quad y \in \mathbb{R}^{m} .
$$

The relations of Algorithm 2.1 can be written as

$$
A V_{m}^{\delta}=V_{m}^{\delta} H_{m}^{\delta}+f_{m+1}^{\delta} e_{m}^{T} .
$$

We refer to (2.5) as an Arnoldi decomposition. It follows from the recursion formulas of the algorithm that

$$
<f_{m+1}^{\delta}, v_{j}^{\delta}>=0, \quad j=1,2, \ldots, m .
$$

Let $\bar{H}_{m}^{\delta} \in \mathbb{R}^{(m+1) \times m}$ be the matrix obtained by appending the row $\left\|f_{m+1}^{\delta}\right\| e_{m}^{T}$ to $H_{m}^{\delta}$. When $f_{m+1}^{\delta} \neq 0$, the Arnoldi decomposition (2.5) can be expressed as

$$
A V_{m}^{\delta}=V_{m+1}^{\delta} \bar{H}_{m}^{\delta},
$$

where $v_{m+1}^{\delta}=f_{m+1}^{\delta} /\left\|f_{m+1}^{\delta}\right\|$. Throughout this paper, we omit the superscript $\delta$ when $\delta=0$.

We now return to the condition (2.3). Assume that the condition is violated. Specifically, let

$$
m-1=\operatorname{dim} \mathcal{K}_{m-1}\left(A, b^{\delta}\right)=\operatorname{dim} \mathcal{K}_{m}\left(A, b^{\delta}\right) .
$$


Then $f_{m}^{\delta}=0$ and Algorithm 2.1 breaks down after the basis $\left\{v_{j}^{\delta}\right\}_{j=1}^{m-1}$ of $\mathcal{K}_{m-1}\left(A, b^{\delta}\right)$ has been determined. Lemma 2.3 below shows that the iterate $x_{m-1}^{\delta}$ computed by the GMRES method solves equation (1.3). This result has been shown by Saad and Schultz [16] for the case $\mathcal{X}=\mathbb{R}^{n}$. We present a proof, because the lemma is important for the development in Section 3. We need the following auxiliary result.

Lemma 2.2. Assume that the operator $A: \mathcal{X} \rightarrow \mathcal{X}$ is invertible on $\mathcal{R}(A)$. Then

$$
\operatorname{dim} A \mathcal{K}_{m}\left(A, b^{\delta}\right)=\operatorname{dim} \mathcal{K}_{m}\left(A, b^{\delta}\right), \quad m=1,2, \ldots,
$$

where $A \mathcal{K}_{m}\left(A, b^{\delta}\right)=\operatorname{span}\left\{A b^{\delta}, A^{2} b^{\delta}, \ldots, A^{m} b^{\delta}\right\}$.

Proof. We have $\operatorname{dim} A \mathcal{K}_{m}\left(A, b^{\delta}\right) \leq \operatorname{dim} \mathcal{K}_{m}\left(A, b^{\delta}\right)$. Assume that $\operatorname{dim} A \mathcal{K}_{m}\left(A, b^{\delta}\right)<\operatorname{dim} \mathcal{K}_{m}\left(A, b^{\delta}\right)$. Then there exists $z \in \mathcal{K}_{m}\left(A, b^{\delta}\right), z \neq 0$, such that $A z=0$. Since $A$ is invertible on $\mathcal{R}(A)$, it follows that $A z=0$ if and only if $z=0$, contradicting the assumption. Thus, $\operatorname{dim} A \mathcal{K}_{m}\left(A, b^{\delta}\right)=\operatorname{dim} \mathcal{K}_{m}\left(A, b^{\delta}\right)$.

Lemma 2.3. Let the operator $A: \mathcal{X} \rightarrow \mathcal{X}$ be invertible on $\mathcal{R}(A)$. Assume that equation (2.7) holds. Then the iterate $x_{m-1}^{\delta}$ determined by the GMRES method applied to equation (1.3) with initial approximate solution $x_{0}^{\delta}=0$ satisfies

$$
A x_{m-1}^{\delta}=b^{\delta} .
$$

Conversely, assume that equation (2.8) holds. Then Algorithm 2.1 breaks down after the orthonormal basis $\left\{v_{j}^{\delta}\right\}_{j=1}^{m-1}$ of $\mathcal{K}_{m-1}\left(A, b^{\delta}\right)$ has been determined.

Proof. Assume that equation (2.7) holds. It then follows from Lemma 2.2 that $b^{\delta} \in A \mathcal{K}_{m-1}\left(A, b^{\delta}\right)$. Thus, there is an element $x_{m-1}^{\delta} \in \mathcal{K}_{m-1}\left(A, b^{\delta}\right)$, such that $b^{\delta}=$ $A x_{m-1}^{\delta}$.

Conversely, assume that equation (2.8) holds. Since $x_{m-1}^{\delta} \in \mathcal{K}_{m-1}\left(A, b^{\delta}\right)$, it follows that $b^{\delta} \in A \mathcal{K}_{m-1}\left(A, b^{\delta}\right)$. But $\mathcal{K}_{m}\left(A, b^{\delta}\right)=\operatorname{span}\left\{b^{\delta}, A \mathcal{K}_{m-1}\left(A, b^{\delta}\right)\right\}$ and therefore $\operatorname{dim} \mathcal{K}_{m}\left(A, b^{\delta}\right)=\operatorname{dim} A \mathcal{K}_{m-1}\left(A, b^{\delta}\right)$. It follows from Lemma 2.2 that $\operatorname{dim} A \mathcal{K}_{m-1}\left(A, b^{\delta}\right)=\operatorname{dim} \mathcal{K}_{m-1}\left(A, b^{\delta}\right)$. Thus, $\operatorname{dim} \mathcal{K}_{m}\left(A, b^{\delta}\right)=\operatorname{dim} \mathcal{K}_{m-1}\left(A, b^{\delta}\right)$.

We point out the following property of Krylov subspaces.

Lemma 2.4. Assume that equation (2.7) holds. Then

$$
\mathcal{K}_{\ell}\left(A, b^{\delta}\right)=\mathcal{K}_{m-1}\left(A, b^{\delta}\right), \quad \ell \geq m .
$$

Proof. Since $\mathcal{K}_{m-1}\left(A, b^{\delta}\right) \subset \mathcal{K}_{\ell}\left(A, b^{\delta}\right)$ for all $\ell \geq m$, it remains to be shown that

$$
A^{j} b^{\delta} \in \mathcal{K}_{m-1}\left(A, b^{\delta}\right), \quad j \geq m .
$$

Equation (2.9) can be shown by induction for increasing values of $j$ using (2.7).

We remark that if $\operatorname{dim} \mathcal{K}_{m}\left(A, b^{\delta}\right)=m$ for all $m \in \mathbb{N}$, then no breakdown of Algorithm 2.1 will occur.

It follows from (2.6) and the fact that $x_{m}^{\delta}=V_{m}^{\delta} y_{m}$ for some $y_{m} \in \mathbb{R}^{m}$, that when $f_{m+1}^{\delta} \neq 0$, the minimization problem (2.2) can be written in the form

$$
\begin{aligned}
\left\|A x_{m}^{\delta}-b^{\delta}\right\| & =\min _{x \in \mathcal{K}_{m}\left(A, b^{\delta}\right)}\left\|A x-b^{\delta}\right\|=\min _{y \in \mathbb{R}^{m}}\left\|A V_{m}^{\delta} y-b^{\delta}\right\| \\
& =\min _{y \in \mathbb{R}^{m}}\left\|V_{m+1}^{\delta}\left(\bar{H}_{m}^{\delta} y-\left\|b^{\delta}\right\| e_{1}\right)\right\| \\
& =\min _{y \in \mathbb{R}^{m}}\left\|\bar{H}_{m}^{\delta} y-\right\| b^{\delta}\left\|e_{1}\right\| .
\end{aligned}
$$

Here and throughout the remainder of this paper $\||\cdot| \mid$ denotes the Euclidean norm on $\mathbb{R}^{m}$ or the associated induced matrix norm. Denote the Moore-Penrose pseudo-inverse 
of $\bar{H}_{m}^{\delta}$ by $\left(\bar{H}_{m}^{\delta}\right)^{\dagger}$. Then the solution of the minimization problem (2.10) is given by $y_{m}=\left\|b^{\delta}\right\|\left(\bar{H}_{m}^{\delta}\right)^{\dagger} e_{1}$, and it follows that

$$
\begin{aligned}
x_{m}^{\delta} & =V_{m}^{\delta}\left(\bar{H}_{m}^{\delta}\right)^{\dagger}\left\|b^{\delta}\right\| e_{1} \\
& =V_{m}^{\delta}\left(\bar{H}_{m}^{\delta}\right)^{\dagger}\left(V_{m+1}^{\delta}\right)^{*} V_{m+1}^{\delta}\left\|b^{\delta}\right\| e_{1} \\
& =V_{m}^{\delta}\left(\bar{H}_{m}^{\delta}\right)^{\dagger}\left(V_{m+1}^{\delta}\right)^{*} b^{\delta},
\end{aligned}
$$

where $\left(V_{m+1}^{\delta}\right)^{*}: \mathcal{X} \rightarrow \mathbb{R}^{m+1}$ denotes the operator adjoint to $V_{m+1}$. It is given by

$$
\left(V_{m+1}^{\delta}\right)^{*} x=\sum_{j=1}^{m+1}<v_{j}^{\delta}, x>e_{j} .
$$

Equation (2.11) yields an explicit representation of the operator $\left(A_{m}^{\delta}\right)^{-1}$ introduced in (1.7) for the GMRES method,

$$
\left(A_{m}^{\delta}\right)^{-1}=V_{m}^{\delta}\left(\bar{H}_{m}^{\delta}\right)^{\dagger}\left(V_{m+1}^{\delta}\right)^{*} .
$$

When $f_{m+1}^{\delta}=0$, the right-hand side of (2.13) simplifies to

$$
\left(A_{m}^{\delta}\right)^{-1}=V_{m}^{\delta}\left(H_{m}^{\delta}\right)^{-1}\left(V_{m}^{\delta}\right)^{*}
$$

Let $\Pi_{m}$ denote the set of polynomials of degree at most $m$, and let $\Pi_{m}^{(0)}=\{p \in$ $\left.\Pi_{m}: p(0)=1\right\}$. The iterate $x_{m}^{\delta} \in \mathcal{K}_{m}\left(A, b^{\delta}\right)$ determined in step $m$ of the GMRES method applied to (1.3) with $x_{0}^{\delta}=0$ can be expressed as

$$
x_{m}^{\delta}=q_{m-1}^{\delta}(A) b^{\delta},
$$

for some $q_{m-1}^{\delta} \in \Pi_{m-1}$, and the associated discrepancy

$$
d_{m}^{\delta}=b^{\delta}-A x_{m}^{\delta}
$$

can be written as

$$
d_{m}^{\delta}=p_{m}^{\delta}(A) b^{\delta},
$$

where

$$
p_{m}^{\delta}(t)=1-t q_{m-1}^{\delta}(t) \in \Pi_{m}^{(0)}
$$

is referred to as the discrepancy polynomial. We remark that in the literature on iterative methods, the quantity $d_{m}^{\delta}$ is often referred to as the residual error and $p_{m}^{\delta}$ as the associated residual polynomial; see, e.g., Saad [15].

In view of (2.2), the discrepancy polynomial $p_{m}^{\delta}$ satisfies

$$
\left\|p_{m}^{\delta}(A) b^{\delta}\right\| \leq\left\|p(A) b^{\delta}\right\|, \quad \forall p \in \Pi_{m}^{(0)} .
$$

We will use this inequality in Section 3. 
3. Regularizing property of the GMRES method. For notational simplicity, we will throughout this section assume that the operator $A$ and right-hand side $b$ in (1.1) are scaled so that

$$
\|A\| \leq 1, \quad\|b\| \leq 1
$$

In this and the following sections, the initial approximate solutions for all iterative methods considered for the solution of equations (1.1) and (1.3) are chosen to be $x_{0}=0$ and $x_{0}^{\delta}=0$, respectively.

The following example illustrates that iterative methods that determine approximate solutions of the equation (1.3) in a sequence of Krylov subspaces (2.1) might not define regularization methods unless additional conditions on the equation are imposed.

Example 3.1. Let $\mathcal{X}=\ell_{2}$ and let $A: \mathcal{X} \rightarrow \mathcal{X}$ be the down-shift operator, i.e., $A\left(\xi_{1}, \xi_{2}, \ldots\right)^{T}=\left(0, \xi_{1}, \xi_{2}, \ldots\right)^{T}$ for $\left(\xi_{1}, \xi_{2}, \ldots\right)^{T} \in \mathcal{X}$. Then $A$ is bounded with null space $\mathcal{N}(A)=\{0\}$. Define the right-hand side $b$ of (1.1) by

$$
x=(1,0,0, \ldots)^{T}, \quad b=A x=(0,1,0, \ldots)^{T} .
$$

Let $\delta>0$ and define $b^{\delta}=b+(0, \delta, 0,0, \ldots)^{T}$. Then $b^{\delta}$ satisfies (1.2) and

$$
\operatorname{dim} \mathcal{K}_{m}\left(A, b^{\delta}\right)=m, \quad \min _{x \in \mathcal{K}_{m}\left(A, b^{\delta}\right)}\left\|b^{\delta}-A x\right\|=1+\delta, \quad m=1,2, \ldots .
$$

Thus, the GMRES method with initial approximate solution $x_{0}^{\delta}=0$ is not a regularization method for this example. Indeed, the GMRES method cannot even determine a solution to the equation (1.1) with the unperturbed right-hand side $b$.

In order to circumvent the difficulties of Example 3.1, we assume that Algorithm 2.1 applied to $\{A, b\}$ breaks down after $\ell$ steps have been carried out, with $1 \leq \ell<\infty$. Thus, the algorithm determines the orthonormal basis $\left\{v_{j}\right\}_{j=1}^{\ell}$ of $\mathcal{K}_{\ell}(A, b)$ as well as elements $f_{j} \in \mathcal{X}, 1 \leq j \leq \ell+1$, such that

$$
\left\{\begin{array}{cc}
f_{j} & \neq 0, \quad 1 \leq j \leq \ell \\
f_{\ell+1} & =0
\end{array}\right.
$$

Lemma 2.3 shows that the iterate $x_{\ell}$ determined by the GMRES method when applied to equation (1.1) with initial approximate solution $x_{0}=0$ solves (1.1). Therefore, the discrepancy polynomial $p_{\ell}$ associated with $x_{\ell}$ satisfies

$$
p_{\ell}(A) b=0,
$$

where $p_{\ell}=p_{\ell}^{\delta}$ for $\delta=0$, cf. (2.15). It follows from (1.7) and (2.14) that the solution $x_{\ell}$ can be written in the form

$$
x_{\ell}=V_{\ell} H_{\ell}^{-1} V_{\ell}^{*} b .
$$

In the remainder of this section, we assume that the positive constant $\alpha$ in Stopping Rule 1.1 satisfies

$$
\alpha \geq\left\|p_{\ell}(A)\right\|
$$

Lemma 3.1. Let $m_{\delta}$ be the termination index determined by Stopping Rule 1.1, with the positive constant $\alpha$ satisfying (3.5), when the GMRES method is applied to equation (1.3) with initial approximate solution $x_{0}^{\delta}=0$. Then $m_{\delta} \leq \ell$. 
Proof. Assume that $m_{\delta}>\ell$ and let $p_{\ell}^{\delta}$ and $p_{\ell}$ be discrepancy polynomials determined by $\ell$ steps of the GMRES method applied to the equations (1.3) and (1.1), respectively. It then follows from (3.5), (2.16) and (1.2) that

$$
\alpha \delta<\left\|p_{\ell}^{\delta}(A) b^{\delta}\right\| \leq\left\|p_{\ell}(A) b^{\delta}\right\|=\left\|p_{\ell}(A)\left(b^{\delta}-b\right)\right\| \leq\left\|p_{\ell}(A)\right\|\left\|b^{\delta}-b\right\| \leq \alpha \delta,
$$

a contradiction. Hence, $m_{\delta} \leq \ell$. $\square$

Assume that $\delta<\|b\|$ and let $b^{\delta} \in \mathcal{X}$ satisfy (1.2). Then $b^{\delta} \neq 0$ and therefore $\operatorname{dim} \mathcal{K}_{\ell}\left(A, b^{\delta}\right) \geq 1$. Let

$$
n=\min \left\{\ell, \operatorname{dim} \mathcal{K}_{\ell}\left(A, b^{\delta}\right)\right\} .
$$

Clearly, the set $\left\{v_{j}^{\delta}\right\}_{j=1}^{n}$ can be determined by Algorithm 2.1 applied to $\left\{A, b^{\delta}\right\}$ with $m=n$. We proceed to show that $n=\ell$ for all $\delta>0$ sufficiently small.

LEMma 3.2. Let $n$ be defined by (3.6), and let $\left\{v_{j}\right\}_{j=1}^{n}$ and $\left\{v_{j}^{\delta}\right\}_{j=1}^{n}$ be orthonormal bases of $\mathcal{K}_{n}(A, b)$ and $\mathcal{K}_{n}\left(A, b^{\delta}\right)$, respectively, determined by Algorithm 2.1 applied to $\{A, b\}$ and $\left\{A, b^{\delta}\right\}$. Then, for $1 \leq j, k \leq n$,

$$
\left\|<A v_{j}^{\delta}, v_{k}^{\delta}>v_{k}^{\delta}-<A v_{j}, v_{k}>v_{k}\right\| \leq 2\left\|v_{k}-v_{k}^{\delta}\right\|+\left\|v_{j}-v_{j}^{\delta}\right\| .
$$

Proof. The inequalities (3.7) follow from application of the Cauchy-Schwarz and triangle inequalities using the bound (3.1) of $A$. $\mathrm{C}$

Define recursively, for $j=1,2, \ldots, \ell+1$, the constants

$$
\mu_{j}= \begin{cases}1, & j=1 \\ \frac{2 j}{\left\|f_{j-1}\right\|} \mu_{j-1}+2 \sum_{k=1}^{j-1} \frac{2}{\left\|f_{k}\right\|} \mu_{k}, & 2 \leq j \leq \ell+1 .\end{cases}
$$

Note that in view of the properties (3.2) of the $f_{j}$, the constants $\mu_{j}$ are well-defined and positive.

Theorem 3.3. Let the sets $\left\{f_{j}\right\}_{j=1}^{n+1}$ and $\left\{v_{j}\right\}_{j=1}^{n}$ be determined by Algorithm 2.1 applied to $\{A, b\}$, and let the sets $\left\{f_{j}^{\delta}\right\}_{j=1}^{n+1}$ and $\left\{v_{j}^{\delta}\right\}_{j=1}^{n}$ be determined by Algorithm 2.1 applied to $\left\{A, b^{\delta}\right\}$, with $m=n$. Introduce

$$
\delta_{j}= \begin{cases}\delta, & j=1, \\ j\left\|v_{j-1}-v_{j-1}^{\delta}\right\|+2 \sum_{k=1}^{j-1}\left\|v_{k}-v_{k}^{\delta}\right\|, & 2 \leq j \leq n+1 .\end{cases}
$$

Then

$$
\begin{aligned}
&\left\|f_{j}-f_{j}^{\delta}\right\| \leq \delta_{j}, \quad 1 \leq j \leq n+1 \\
&\left\|v_{j}-v_{j}^{\delta}\right\| \leq \frac{2}{\left\|f_{j}\right\|} \delta_{j}, \quad 1 \leq j \leq n .
\end{aligned}
$$

Moreover,

$$
\delta_{j} \leq \mu_{j} \delta, \quad 1 \leq j \leq n+1,
$$

where the constants $\mu_{j}$ are defined by (3.8).

Proof. We first show the inequalities (3.10). It follows from (1.2) that $\left\|f_{1}-f_{1}^{\delta}\right\|=$ $\left\|b-b^{\delta}\right\| \leq \delta=\delta_{1}$. When $2 \leq j \leq n+1$, it follows from the scaling (3.1) of $A$, the 
formulas for $f_{j}$ and $f_{j}^{\delta}$ in Algorithm 2.1 and the inequalities (3.7) that

$$
\begin{aligned}
\left\|f_{j}-f_{j}^{\delta}\right\| & =\left\|A v_{j-1}-\sum_{k=1}^{j-1}<A v_{j-1}, v_{k}>v_{k}-A v_{j-1}^{\delta}+\sum_{k=1}^{j-1}<A v_{j-1}^{\delta}, v_{k}^{\delta}>v_{k}^{\delta}\right\| \\
& \leq\left\|A v_{j-1}-A v_{j-1}^{\delta}\right\|+\left\|\sum_{k=1}^{j-1}\left(<A v_{j-1}^{\delta}, v_{k}^{\delta}>v_{k}^{\delta}-<A v_{j-1}, v_{k}>v_{k}\right)\right\| \\
& \leq\left\|v_{j-1}-v_{j-1}^{\delta}\right\|+\sum_{k=1}^{j-1}\left(2\left\|v_{k}-v_{k}^{\delta}\right\|+\left\|v_{j-1}-v_{j-1}^{\delta}\right\|\right) \\
& =j\left\|v_{j-1}-v_{j-1}^{\delta}\right\|+2 \sum_{k=1}^{j-1}\left\|v_{k}-v_{k}^{\delta}\right\|=\delta_{j} .
\end{aligned}
$$

We turn to the inequalities (3.11). The relations $v_{j}=f_{j} /\left\|f_{j}\right\|$ and $v_{j}^{\delta}=f_{j}^{\delta} /\left\|f_{j}^{\delta}\right\|$ yield

$$
\begin{aligned}
\left\|v_{j}-v_{j}^{\delta}\right\| & =\left\|\frac{f_{j}}{\left\|f_{j}\right\|}-\frac{f_{j}^{\delta}}{\left\|f_{j}^{\delta}\right\|}\right\|=\left\|\frac{\left(\left\|f_{j}^{\delta}\right\|-\left\|f_{j}\right\|\right) f_{j}^{\delta}+\left\|f_{j}^{\delta}\right\|\left(f_{j}-f_{j}^{\delta}\right)}{\left\|f_{j}\right\|\left\|f_{j}^{\delta}\right\|}\right\| \\
& \leq \frac{\left|\left\|f_{j}^{\delta}\right\|-\left\|f_{j}\right\|\right|}{\left\|f_{j}\right\|}+\frac{\left\|f_{j}-f_{j}^{\delta}\right\|}{\left\|f_{j}\right\|} \leq \frac{2}{\left\|f_{j}\right\|}\left\|f_{j}-f_{j}^{\delta}\right\|, \quad 1 \leq j \leq n .
\end{aligned}
$$

Combining these inequalities with (3.10) establishes (3.11).

We are now in a position to show (3.12). It follows from the inequalities (3.11) and the definitions (3.9) of the $\delta_{j}$ and (3.8) of the $\mu_{j}$ that $\delta_{1} \leq \mu_{1} \delta$ and

$$
\delta_{j} \leq \frac{2 j}{\left\|f_{j-1}\right\|} \mu_{j-1} \delta+2 \sum_{k=1}^{j-1} \frac{2}{\left\|f_{k}\right\|} \mu_{k} \delta=\mu_{j} \delta, \quad 2 \leq j \leq n+1 .
$$

Corollary 3.4. Let $\mu_{j}, 1 \leq j \leq \ell$, be given by (3.8) and define the positive constant

$$
\hat{\delta}=\min _{1 \leq j \leq \ell}\left\{\frac{\left\|f_{j}\right\|}{2 \mu_{j}}\right\} .
$$

Then $\operatorname{dim} \mathcal{K}_{\ell}\left(A, b^{\delta}\right)=\ell$ for all $b^{\delta} \in \mathcal{X}$ such that $\left\|b-b^{\delta}\right\| \leq \hat{\delta}$.

Proof. Assume that there is a $b^{\delta} \in \mathcal{X}$, such that $\left\|b-b^{\delta}\right\| \leq \hat{\delta}$ and $n=$ $\operatorname{dim} \mathcal{K}_{\ell}\left(A, b^{\delta}\right)<\ell$. In view of $(3.2)$, the element $f_{n+1} \in \mathcal{X}$ determined by Algorithm 2.1 when applied to $\{A, b\}$ is nonvanishing. Moreover, Algorithm 2.1 applied to $\left\{A, b^{\delta}\right\}$ with $m=n$ yields $f_{j}^{\delta} \neq 0$ for $1 \leq j \leq n$ and $f_{n+1}^{\delta}=0$. But application of (3.13), (3.12) and (3.10), in order, yields

$$
\frac{\left\|f_{n+1}\right\|}{2}=\frac{\mu_{n+1}\left\|f_{n+1}\right\|}{2 \mu_{n+1}} \geq \mu_{n+1} \hat{\delta} \geq \delta_{n+1} \geq\left\|f_{n+1}-f_{n+1}^{\delta}\right\|=\left\|f_{n+1}\right\|
$$

a contradiction, because $\left\|f_{n+1}\right\| \neq 0$. We conclude that $\operatorname{dim} \mathcal{K}_{\ell}\left(A, b^{\delta}\right)=\ell$ for all $b^{\delta}$ such that $\left\|b-b^{\delta}\right\| \leq \hat{\delta}$.

LEMMA 3.5. Let $\delta$ satisfy $0<\delta \leq \hat{\delta}$, where $\hat{\delta}$ is defined by (3.13), and let $b^{\delta} \in \mathcal{X}$ satisfy (1.2). Let the operators $V_{k}$ and $V_{k}^{\delta}$ be determined by applying $k \leq \ell$ steps of Algorithm 2.1 to $\{A, b\}$ and $\left\{A, b^{\delta}\right\}$, respectively; cf. (2.4). Then

$$
\left\|V_{k}-V_{k}^{\delta}\right\| \leq \beta_{k} \delta, \quad 1 \leq k \leq \ell,
$$


where

$$
\beta_{k}=\sum_{j=1}^{k} \frac{2}{\left\|f_{j}\right\|} \mu_{j},
$$

and the $\mu_{j}$ are given by (3.8). Furthermore, the adjoint operators $\left(V_{k}^{\delta}\right)^{*}$ satisfy

$$
\left\|V_{k}^{*}-\left(V_{k}^{\delta}\right)^{*}\right\| \leq \beta_{k} \delta, \quad 1 \leq k \leq \ell .
$$

The norms $\|\cdot\|$ in (3.14) and (3.16) are the operator norms induced by the norms $\|\cdot\|$ on $\mathbb{R}^{k}$ and $\|\cdot\|$ on $\mathcal{X}$.

Proof. Let $1 \leq k \leq \ell$. By Corollary $3.4, \operatorname{dim} \mathcal{K}_{k}\left(A, b^{\delta}\right)=k$ for any $b^{\delta} \in \mathcal{X}$ with $\left\|b-b^{\delta}\right\| \leq \hat{\delta}$. Therefore $k$ steps of Algorithm 2.1 can be carried out without breakdown and the orthonormal basis $\left\{v_{j}^{\delta}\right\}_{j=1}^{k}$ of $\mathcal{K}_{k}\left(A, b^{\delta}\right)$ determined by the algorithm is welldefined. Thus, the operators $V_{k}$ and $V_{k}^{\delta}$, defined by (2.4) with $m=k$, exist. The bounds (3.11) and (3.12) yield

$$
\begin{aligned}
\left\|V_{k}-V_{k}^{\delta}\right\| & =\sup _{\substack{y \in \mathbb{R}^{k} \\
\|y\|=1}}\left\|\left(V_{k}-V_{k}^{\delta}\right) y\right\| \leq \sup _{\substack{y \in \mathbb{R}^{k} \\
\|y\|=1}} \sum_{j=1}^{k}\left|e_{j}^{T} y\right|\left\|v_{j}-v_{j}^{\delta}\right\| \\
& \leq \sum_{j=1}^{k}\left\|v_{j}-v_{j}^{\delta}\right\| \leq \sum_{j=1}^{k} \frac{2}{\left\|f_{j}\right\|} \mu_{j} \delta
\end{aligned}
$$

and (3.14) follows. The operator $V_{k}^{*}-\left(V_{k}^{\delta}\right)^{*}$ is the adjoint of $V_{k}-\left(V_{k}^{\delta}\right)$. Therefore $\left\|V_{k}^{*}-\left(V_{k}^{\delta}\right)^{*}\right\|=\left\|V_{k}-\left(V_{k}^{\delta}\right)\right\|$ and the inequality (3.16) follows from (3.14).

Lemma 3.6. Let $\delta$ satisfy $0<\delta \leq \hat{\delta}$, where $\hat{\delta}$ is defined by (3.13), and let $b^{\delta} \in \mathcal{X}$ satisfy (1.2). Let the matrices $\bar{H}_{k}^{\delta} \in \mathbb{R}^{(k+1) \times k}$ and $\bar{H}_{k} \in \mathbb{R}^{(k+1) \times k}$ be determined by $k \leq \ell$ steps of Algorithm 2.1 applied to $\left\{A, b^{\delta}\right\}$ and $\{A, b\}$, respectively, cf. (2.6). Let $\beta_{k}$ be defined by (3.15) and $\mu_{k+1}$ by (3.8). Then the matrices $\bar{H}_{k}$ and $\bar{H}_{k}^{\delta}$ satisfy

$$
\left\|\bar{H}_{k}-\bar{H}_{k}^{\delta}\right\| \leq\left(2 \beta_{k}+\mu_{k+1}\right) \delta, \quad 1 \leq k \leq \ell .
$$

Proof. It follows from Corollary 3.4 that the matrices $\bar{H}_{k}$ and $\bar{H}_{k}^{\delta}$ exist. The inequalities (3.10) and (3.12) with $j=k+1$ can be used to establish the inequality

$$
\left\|\bar{H}_{k}-\bar{H}_{k}^{\delta}\right\|\left|\leq\left\|H_{k}-H_{k}^{\delta}\right\|\right|+\left\|f_{k+1}-f_{k+1}^{\delta}\right\| \leq\left\|H_{k}-H_{k}^{\delta}\right\| \mid+\mu_{k+1} \delta .
$$

It follows from (2.5) with $m=k$ that $H_{k}^{\delta}=\left(V_{k}^{\delta}\right)^{*} A V_{k}^{\delta}$, and similarly $H_{k}=V_{k}^{*} A V_{k}$. These identities, the scaling (3.1) of $A$, the fact that $\left\|V_{k}\right\|=\left\|V_{k}^{\delta}\right\|=1$, and the inequalities (3.14) and (3.16) yield

$$
\begin{aligned}
\left\|H_{k}-H_{k}^{\delta}\right\| & =\left\|V_{k}^{*} A V_{k}-\left(V_{k}^{\delta}\right)^{*} A V_{k}^{\delta}\right\| \| \\
& \leq\left\|\left(V_{k}^{*}-\left(V_{k}^{\delta}\right)^{*}\right) A V_{k}+\left(V_{k}^{\delta}\right)^{*} A\left(V_{k}-V_{k}^{\delta}\right)\right\| \| \\
& \leq\left\|V_{k}^{*}-\left(V_{k}^{\delta}\right)^{*}\right\|+\left\|V_{k}-V_{k}^{\delta}\right\| \leq 2 \beta_{k} \delta .
\end{aligned}
$$

Combining the inequalities (3.18) and (3.19) completes the proof.

The following results discuss the sensitivity of the pseudo-inverse of a matrix to perturbations. 
Lemma 3.7. (Björck [2, Theorem 1.4.2]) Let $B, E \in \mathbb{R}^{k \times j}$ satisfy $\operatorname{rank}(B+E)=$ $\operatorname{rank}(B)$ and $\eta=\left\|B^{\dagger}\right\||\|||E| \mid<1$. Then

$$
\left\|(B+E)^{\dagger}\right\| \mid \leq \frac{1}{1-\eta}\left\|B^{\dagger}\right\| .
$$

Lemma 3.8. Let the matrices $\bar{H}_{k}$ and $\bar{H}_{k}^{\delta}$ as well as the constants $\beta_{k}, \mu_{k+1}$ and $\hat{\delta}$ be defined as in Lemma 3.6, and introduce the positive constants

$$
\gamma=\max _{1 \leq k \leq \ell}\left\{\left\|\bar{H}_{k}^{\dagger}\right\| \mid\left(2 \beta_{k}+\mu_{k+1}\right)\right\}
$$

and

$$
\tilde{\delta}=\min \left\{\frac{1}{2 \gamma}, \hat{\delta}\right\}
$$

Let $\delta$ satisfy $0<\delta \leq \tilde{\delta}$ and assume that $b^{\delta} \in \mathcal{X}$ satisfies (1.2). Then

$$
\left\|\left(\bar{H}_{k}^{\delta}\right)^{\dagger}\right\| \leq 2\left\|\bar{H}_{k}^{\dagger}\right\|, \quad 1 \leq k \leq \ell .
$$

Moreover, when $f_{k+1}^{\delta} \neq 0$, we have that

$$
\left\|\left(\bar{H}_{k}^{\delta}\right)^{\dagger}-\bar{H}_{k}^{\dagger}\right\|\left|\leq 2^{3 / 2}\left\|\bar{H}_{k}^{\dagger}\right\|\right|^{2}\left(2 \beta_{k}+\mu_{k+1}\right) \delta .
$$

Proof. Let $k$ satisfy $1 \leq k \leq \ell$. It follows from Corollary 3.4 that the matrices used in this lemma exist. We first show (3.22). Inequality (3.17) yields

$$
\left\|\left|\bar{H}_{k}^{\dagger}\left\|\left|\left\||| \bar{H}_{k}-\bar{H}_{k}^{\delta}\right\|\right| \leq\right\| \bar{H}_{k}^{\dagger} \|\right|\left(2 \beta_{k}+\mu_{k+1}\right) \delta \leq \gamma \tilde{\delta} \leq \frac{1}{2},\right.
$$

and by Corollary 3.4, we have

$$
\operatorname{rank}\left(\bar{H}_{k}\right)=\operatorname{rank}\left(\bar{H}_{k}^{\delta}\right)=k .
$$

Lemma 3.7 can now be applied to show that

$$
\left\|\left|\left(\bar{H}_{k}^{\delta}\right)^{\dagger}\left\|\left|\leq \frac{1}{1-\left\|\bar{H}_{k}^{\dagger}\right\|\left|\left\||| \bar{H}_{k}-\bar{H}_{k}^{\delta}\right\| \|\right.}\left\|\bar{H}_{k}^{\dagger}\right\|\right| \leq \frac{1}{1-\gamma \tilde{\delta}}\right\| \bar{H}_{k}^{\dagger}\|\mid \leq 2\| \bar{H}_{k}^{\dagger}\|\| .\right.\right.
$$

We turn to (3.23). This inequality can be shown by using (3.22), (3.24) and

$$
\left\|\left|\bar{H}_{k}^{\dagger}-\left(\bar{H}_{k}^{\delta}\right)^{\dagger}\left\|\left|\leq 2^{1 / 2}\left\|\left|\overline { H } _ { k } ^ { \dagger } \left\|| | \left|\bar{H}_{k}^{\delta}-\bar{H}_{k}\left\||| \mid\left(\bar{H}_{k}^{\delta}\right)^{\dagger}\right\| .\right.\right.\right.\right.\right.\right.\right.\right.
$$

The latter inequality is due to Wedin [17].

THEOREM 3.9. Let $\delta$ satisfy $0<\delta \leq \tilde{\delta}$, where $\tilde{\delta}$ is given by (3.21), and let $b^{\delta} \in \mathcal{X}$ satisfy (1.2). Let $k \leq \ell$ and let $x_{k}^{\delta}$ denote the $k$ th iterate determined by the GMRES method applied to equation (1.3) with initial approximate solution $x_{0}^{\delta}=0$. Similarly, let $x_{k}$ denote the $k$ th iterate determined by the GMRES method applied to equation (1.1) with initial approximate solution $x_{0}=0$. Then, there are constants $\sigma_{k}$ independent of $\delta$, such that

$$
\left\|x_{k}-x_{k}^{\delta}\right\| \leq \sigma_{k} \delta, \quad 1 \leq k \leq \ell .
$$


Proof. It follows from Corollary 3.4 that $\operatorname{dim} \mathcal{K}_{\ell}\left(A, b^{\delta}\right)=\ell$ and therefore it can be seen from Lemma 2.3 that, for $k \leq \ell$, the approximate solution $x_{k}^{\delta}$ determined by the GMRES method applied to (1.3) is well-defined. Introduce

$$
\hat{v}_{\ell+1}^{\delta}= \begin{cases}f_{\ell+1}^{\delta} /\left\|f_{\ell+1}^{\delta}\right\|, & \text { if }\left\|f_{\ell+1}^{\delta}\right\|>0 \\ 0, & \text { if }\left\|f_{\ell+1}^{\delta}\right\|=0\end{cases}
$$

and let the operators $\left(\hat{V}_{\ell+1}^{\delta}\right)^{*}: \mathcal{X} \rightarrow \mathbb{R}^{\ell+1}$ and $\left(\hat{V}_{\ell+1}\right)^{*}: \mathcal{X} \rightarrow \mathbb{R}^{\ell+1}$ be defined by

$$
\left\{\begin{aligned}
\left(\hat{V}_{\ell+1}^{\delta}\right)^{*} x & =\sum_{j=1}^{\ell}<v_{j}^{\delta}, x>e_{j}+<\hat{v}_{\ell+1}^{\delta}, x>e_{\ell+1}, \\
\hat{V}_{\ell+1}^{*} x & =\sum_{j=1}^{\ell}<v_{j}, x>e_{j}+<\hat{v}_{\ell+1}^{\delta}, x>e_{\ell+1} .
\end{aligned}\right.
$$

The expression (2.11) yields

$$
x_{k}-x_{k}^{\delta}=\left\{\begin{array}{l}
V_{k} \bar{H}_{k}^{\dagger} V_{k+1}^{*} b-V_{k}^{\delta}\left(\bar{H}_{k}^{\delta}\right)^{\dagger}\left(V_{k+1}^{\delta}\right)^{*} b^{\delta}, \text { if } k<\ell, \\
V_{k} \bar{H}_{k}^{\dagger} \hat{V}_{k+1}^{*} b-V_{k}^{\delta}\left(\bar{H}_{k}^{\delta}\right)^{\dagger}\left(\hat{V}_{k+1}^{\delta}\right)^{*} b^{\delta}, \text { if } k=\ell .
\end{array}\right.
$$

When $k<\ell$, it follows from the bounds (3.14), (3.16), (3.22) and (3.23), the scaling (3.1) of $b$, and the fact that $\left\|V_{j}^{\delta}\right\|=\left\|\left(V_{j}^{\delta}\right)^{*}\right\|=1$ for $1 \leq j \leq \ell$ and all $\delta \geq 0$, that

$$
\begin{aligned}
\left\|x_{k}-x_{k}^{\delta}\right\|= & \left\|V_{k} \bar{H}_{k}^{\dagger} V_{k+1}^{*} b-V_{k}^{\delta}\left(\bar{H}_{k}^{\delta}\right)^{\dagger}\left(V_{k+1}^{\delta}\right)^{*} b^{\delta}\right\| \\
= & \|\left(V_{k}-V_{k}^{\delta}\right) \bar{H}_{k}^{\dagger} V_{k+1}^{*} b+V_{k}^{\delta}\left(\bar{H}_{k}^{\dagger}-\left(\bar{H}_{k}^{\delta}\right)^{\dagger}\right)\left(V_{k+1}^{\delta}\right)^{*} b^{\delta} \\
& +V_{k}^{\delta} \bar{H}_{k}^{\dagger}\left(V_{k+1}^{*}-\left(V_{k+1}^{\delta}\right)^{*}\right) b+V_{k}^{\delta} \bar{H}_{k}^{\dagger}\left(V_{k+1}^{\delta}\right)^{*}\left(b-b^{\delta}\right) \| \\
\leq & \left\|V_{k}-V_{k}^{\delta}\right\|\|\| \bar{H}_{k}^{\dagger}\|+\|+\left\|\bar{H}_{k}^{\dagger}-\left(\bar{H}_{k}^{\delta}\right)^{\dagger}\right\|(1+\delta) \\
& +\left\|\bar{H}_{k}^{\dagger}\right\|\left\|V_{k+1}^{*}-\left(V_{k+1}^{\delta}\right)^{*}\right\|+\left\|\bar{H}_{k}^{\dagger}\right\| \delta \\
\leq & \beta_{k} \delta\left\|\bar{H}_{k}^{\dagger}\right\|\left|+2\left\|\bar{H}_{k}^{\dagger}\right\|\right|^{2}\left(2 \beta_{k}+\mu_{k+1}\right) \delta(1+\delta)+\left\|\bar{H}_{k}^{\dagger}\right\| \beta_{k+1} \delta+\left\|\bar{H}_{k}^{\dagger}\right\| \| \delta \\
= & 2\left(2 \beta_{k}+\mu_{k+1}\right)\left\|\bar{H}_{k}^{\dagger}\right\|^{2}(1+\delta) \delta+\left(\beta_{k}+\beta_{k+1}+1\right)\left\|\bar{H}_{k}^{\dagger}\right\| \delta .
\end{aligned}
$$

Thus, we can choose

$$
\sigma_{k}=2\left(2 \beta_{k}+\mu_{k+1}\right)\left\|\left|\bar{H}^{\dagger}\left\|\left.\right|^{2}(1+\tilde{\delta})+\left(\beta_{k}+\beta_{k+1}+1\right)\right\|\right| \bar{H}_{k}^{\dagger}\right\| .
$$

A similar bound can be shown for $k=\ell$ by using the operators (3.26) and the fact that $\left\|\hat{V}_{\ell+1}^{*}-\left(\hat{V}_{\ell+1}^{\delta}\right)^{*}\right\| \leq \beta_{\ell} ;$ cf. (3.16).

Introduce the constant

$$
\epsilon=\min _{1 \leq j<\ell} \frac{\left\|b-A x_{j}\right\|}{2\left(\alpha+\sigma_{j}+1\right)}
$$

where $\alpha$ satisfies (3.5) and the $\sigma_{j}, 1 \leq j<\ell$, are as in Theorem 3.9. Note that by Lemma 2.3, $\left\|b-A x_{k}\right\|>0$ for $1 \leq k<\ell$, and therefore $\epsilon>0$.

THEOREM 3.10. Let $\delta$ satisfy $0<\delta \leq \epsilon$, with $\epsilon$ defined by (3.27), and let $b^{\delta} \in \mathcal{X}$ satisfy (1.2). Apply the GMRES method, using Stopping Rule 1.1 with the positive constant $\alpha$ satisfying (3.5), to the solution of equation (1.3) with initial approximate solution $x_{0}^{\delta}=0$. Then the termination index $m_{\delta}$ satisfies $m_{\delta}=\ell$, where $\ell$ is the number of GMRES steps required to solve (1.1) with initial approximate solution $x_{0}=$ 0 . 
Proof. Assume that $m_{\delta} \neq \ell$. It follows from Lemma 3.1 that $m_{\delta}<\ell$, i.e, $\left\|b^{\delta}-A x_{k}^{\delta}\right\| \leq \alpha \delta$ for some $k<\ell$. Theorem 3.9 and the scaling (3.1) of $A$ yield

$$
\alpha \delta \geq\left\|b^{\delta}-A x_{k}^{\delta}\right\| \geq\left\|b-A x_{k}\right\|-\left\|A x_{k}-A x_{k}^{\delta}\right\|-\left\|b-b^{\delta}\right\| \geq\left\|b-A x_{k}\right\|-\sigma_{k} \delta-\delta
$$

and, therefore,

$$
\left\|b-A x_{k}\right\| \leq\left(\alpha+\sigma_{k}+1\right) \delta .
$$

It follows from $\delta \leq \epsilon$ and (3.27) that

$$
\left\|b-A x_{k}\right\| \leq\left(\alpha+\sigma_{k}+1\right) \epsilon \leq \frac{1}{2}\left\|b-A x_{k}\right\|
$$

a contradiction since $\left\|b-A x_{k}\right\|>0$. We conclude that $m_{\delta}=\ell$.

THEOREM 3.11. Assume that Algorithm 2.1 applied to $\{A, b\}$ breaks down after $\ell$ steps, and let the right-hand side $b^{\delta}$ of equation (1.3) satisfy the same conditions as in Theorem 3.10. Let the GMRES method applied to (1.3), using Stopping Rule 1.1 with the positive constant $\alpha$ satisfying (3.5), determine the approximate solution $x_{m_{\delta}}^{\delta}$, where, as usual, the initial approximate solution is chosen to be $x_{0}^{\delta}=0$. Then

$$
\lim _{\delta \searrow 0} \sup _{\left\|b-b^{\delta}\right\| \leq \delta}\left\|x-x_{m_{\delta}}^{\delta}\right\|=0
$$

where $x$ denotes the solution of (1.1).

Proof. It follows from Lemma 2.3 that the $\ell$ th iterate $x_{\ell}$ determined by the GMRES method applied to equation (1.1) with initial approximate solution $x_{0}=0$ solves the equation, i.e., $x_{\ell}=x$. Theorem 3.10 and the bound (3.25) show that $\left\|x-x_{m_{\delta}}^{\delta}\right\|=\left\|x-x_{\ell}^{\delta}\right\| \leq \sigma_{\ell} \delta$, and we obtain

$$
\sup _{\left\|b-b^{\delta}\right\| \leq \delta}\left\|x-x_{m_{\delta}}^{\delta}\right\| \leq \sigma_{\ell} \delta
$$

Letting $\delta$ decrease to zero establishes (3.28).

It follows from Theorem 3.11 that if the GMRES method solves equation (1.1) in finitely many steps, then the GMRES method equipped with Stopping Rule 1.1 defines a regularization method.

We remark that the results of this section also hold when $A$ is singular, provided that equation (1.1) has at least one solution and the GMRES method can compute a specific solution $\breve{x}$ in finitely many steps. Discussions on the behavior of the GMRES method when applied to linear systems of equations with a singular matrix are presented in $[3,4]$. Here we only note that a difficulty that arises is that the Arnoldi process may break down before a sufficiently large Krylov subspace has been generated to contain the solution $\breve{x}$, i.e., the conclusion of Lemma 2.3 may be violated.

4. Computed examples. This section illustrates the behavior of the limit (1.8) when the GMRES method is applied to two problems from the package Regularization Tools by Hansen [10]. We compare the results of the GMRES method to those of the conjugate gradient method applied to the normal equations (1.6). We use the implementation CGLS of Björck [2]. This implementation does not require the operator $A^{*} A$ to be formed explicitly. All computations were performed on an Intel Pentium workstation with about 16 significant decimal digits using GNU Octave. 


\begin{tabular}{|c|c|c|c|c|}
\hline & \multicolumn{2}{|c|}{ Example 4.1} & \multicolumn{3}{|c|}{ Example 4.2} \\
$\delta$ & $m_{\delta}$ (GMRES) & $m_{\delta}$ (CGLS) & $m_{\delta}$ (GMRES) & $m_{\delta}$ (CGLS) \\
\hline $1 \cdot 10^{-1}$ & 11 & 2 & 1 & 2 \\
$1 \cdot 10^{-2}$ & 32 & 7 & 3 & 3 \\
$1 \cdot 10^{-3}$ & 58 & 13 & 3 & 3 \\
$1 \cdot 10^{-4}$ & 90 & 20 & 3 & 4 \\
$1 \cdot 10^{-5}$ & 98 & 26 & 5 & 6 \\
$1 \cdot 10^{-6}$ & 99 & 41 & 5 & 6 \\
$1 \cdot 10^{-7}$ & 100 & 55 & 5 & 7 \\
$1 \cdot 10^{-8}$ & 100 & 79 & 5 & 8 \\
$1 \cdot 10^{-9}$ & 100 & 94 & 6 & 8 \\
$1 \cdot 10^{-10}$ & 100 & 98 & 7 & 8 \\
$1 \cdot 10^{-11}$ & 100 & 98 & 7 & 9 \\
$1 \cdot 10^{-12}$ & 100 & 98 & 7 & 9 \\
\hline
\end{tabular}

Termination index $m_{\delta}$ for different values of $\delta$ for Examples 4.1 and 4.2 for the GMRES and CGLS methods equipped with Stopping Rule 1.1 with $\alpha=1$.

Example 4.1. Consider the Volterra integral equation of the first kind,

$$
\int_{0}^{\tau} \kappa(\tau-\sigma) x(\sigma) d \sigma=b(\tau), \quad 0 \leq \tau \leq 1,
$$

where the kernel is defined by

$$
\kappa(\tau)=\frac{\tau^{-3 / 2}}{2 \sqrt{\pi}} \exp \left(-1 /\left(4 \tau^{2}\right)\right) .
$$

This equation is discussed by Carasso [6]. Let the right-hand side function $b(\tau)$ be chosen so that

$$
x(\tau)= \begin{cases}75 \tau^{2}, & 0 \leq \tau \leq 1 / 10 \\ \frac{3}{4}+(20 \tau-2)(3-20 \tau), & 1 / 10<\tau \leq 3 / 20, \\ \frac{3}{4} \exp (2(3-20 \tau)), & 3 / 20<\tau \leq 1 / 2, \\ 0, & 1 / 2<\tau \leq 1\end{cases}
$$

solves (4.1). We discretize (4.1) by the composite midpoint rule with 100 equidistant nodes in $[0,1]$ using the Matlab code provided by Hansen [10] and obtain a linear system of equations $A x=b$, where $A \in \mathbb{R}^{100 \times 100}$ and $x, b \in \mathbb{R}^{100}$. The matrix $A$ is of ill-determined rank, because its singular values "cluster" at the origin. The vector $x$ is a tabulation of the solution (4.2) at equidistant points.

Let the vector $d \in \mathbb{R}^{100}$ consist of random entries that are uniformly distributed in the interval $[0,1]$. We refer to the vector $d$ as noise. Introduce the perturbed right-hand side vector $b^{\delta}=b+\delta d$, where $\delta>0$.

We apply the GMRES method with initial approximate solution $x_{0}^{\delta}=0$ to the solution of the linear system of equations $A x=b^{\delta}$ for right-hand sides associated with several values of $\delta$. The iterations are terminated when Stopping Rule 1.1 with $\alpha=1$ is satisfied. The Arnoldi process has been implemented with reorthogonalization to secure orthogonality of the computed Krylov subspace basis. We denote the computed approximate solutions by $x_{m_{\delta}}^{\delta}$. Figure 4.1 displays the points $\left\{\log _{10}(\delta), \log _{10}(\| x-\right.$ 
$\left.\left.x_{m_{\delta}}^{\delta}\|/\| x \|\right)\right\}$, marked by “+”, for $\delta \in\left\{1 \cdot 10^{-j}\right\}_{j=1}^{12}$. Adjacent points are connected by dashed straight lines for clarity. The figure shows the convergence of the computed iterates $x_{m_{\delta}}^{\delta}$ to the solution $x$ of equation (1.1) as a function of $\delta$. The termination indices $m_{\delta}$ for the GMRES method determined by Stopping Rule 1.1 for different values of $\delta$ are shown in Table 4.1 .

Figure 4.1 also shows the corresponding graph for iterates $x_{m_{\delta}}^{\delta}$ determined by the CGLS method. In order to reduce the effect of round-off errors on the computed iterates, we implemented the CGLS method with reorthogonalization of the residual vectors $r_{j}^{\delta}=A^{*} b^{\delta}-A^{*} A x_{j}^{\delta}$ associated with the normal equations (1.6) by the modified Gram-Schmidt method. The points $\left\{\log _{10}(\delta), \log _{10}\left(\left\|x-x_{m_{\delta}}^{\delta}\right\| /\|x\|\right)\right\}$ determined by the CGLS method are marked by "o" and adjacent points are connected by continuous straight lines. The termination indices $m_{\delta}$ for the CGLS method determined by Stopping Rule 1.1 for different values of $\delta$ are shown in Table 4.1.

Example 4.2. We consider the Fredholm integral equation of the first kind,

$$
\int_{0}^{\pi / 2} \kappa(\sigma, \tau) x(\sigma) d \sigma=b(\tau), \quad 0 \leq \tau \leq \pi
$$

where $\kappa(\sigma, \tau)=\exp (\sigma \cos (\tau))$ and $b(\tau)=2 \sinh (\tau) / \tau$, discussed by Baart [1]. The solution is given by $x(\tau)=\sin (\tau)$. We use Matlab code from [10] to discretize (4.3) by the Galerkin method with 100 orthonormal box functions as test and trial functions. This gives a linear system of equations $A x=b$, where $A \in \mathbb{R}^{100 \times 100}$ and $x, b \in$ $\mathbb{R}^{100}$. The singular values of $A$ "cluster" at the origin; the matrix therefore is of ill-determined rank. We determine a "noise-vector" $d$ and a "noisy" right-hand side $b^{\delta}$ similarly as in Example 4.1. We solve the linear system of equations $A x^{\delta}=b^{\delta}$ by the GMRES and CGLS methods equipped with Stopping Rule 1.1 for several values of $\delta$ and $\alpha=1$. Figure 4.2 is analogous to Figure 4.1 and displays the 10-logarithm of the relative error $\left\|x-x_{m_{\delta}}^{\delta}\right\| /\|x\|$ as a function of $\delta$ for iterates $x_{m_{\delta}}^{\delta}$ determined by the GMRES and CGLS methods with $\delta \in\left\{1 \cdot 10^{-j}\right\}_{j=1}^{12}$. The termination indices $m_{\delta}$ determined by Stopping Rule 1.1 are shown in Table 4.1 .

The above examples show the iterates $x_{m_{\delta}}^{\delta}$ determined by the GMRES method converge to the solution $x$ of (1.1) as $\delta$ approaches zero for two ill-posed problems from [10]. Note that the relative error $\left\|x-x_{m_{\delta}}^{\delta}\right\| /\|x\|$ in Example 4.2 is considerably smaller for small values of $\delta$ for iterates $x_{m_{\delta}}^{\delta}$ determined by the GMRES method than for iterates computed by the CGLS method.

Further comparisons of the GMRES and CGLS methods are reported in [5], where we consider ill-posed problems (1.3) that arise in image restoration. The computed examples there show the GMRES method applied to equation (1.3) require fewer evaluations of matrix-vector products with the matrices $A$ or $A^{T}$ and determine more pleasing restored images than the CGLS method applied to the associated normal equations (1.6).

Acknowledgement. We would like to thank a referee for comments.

\section{REFERENCES}

[1] M. L. Baart, The use of auto-correlation for pseudo-rank determination in noisy ill-conditioned least-squares problems, IMA J. Numer. Anal., 2 (1982), pp. 241-247.

[2] Å. Björck, Numerical Methods for Least Squares Problems, SIAM, Philadelphia, 1996.

[3] P. N. Brown and H. F. Walker, GMRES on (nearly) singular systems, SIAM J. Matrix Anal. Appl., 18 (1997), pp. 37-51. 
[4] D. Calvetti, B. Lewis and L. Reichel, GMRES-type methods for inconsistent systems, Linear Algebra Appl., 316 (2000), pp. 157-169.

[5] D. Calvetti, B. Lewis and L. Reichel, Restoration of images with spatially variant blur by the GMRES method, in Advanced Signal Processing Algorithms, Architectures, and Implementations X, ed. F. T. Luk, Proceedings of the Society of Photo-Optical Instrumentation Engineers (SPIE), vol. 4116, The International Society for Optical Engineering, Bellingham, WA, 2000, pp. 364-374.

[6] A. S. Carasso, Determining surface temperatures from interior observations, SIAM J. Appl. Math., 42 (1982), pp. 558-574.

[7] C. W. Groetsch, The Theory of Tikhonov Regularization for Fredholm Equations of the First Kind, Pitman, London, 1984.

[8] M. Hanke, Conjugate Gradient Type Methods for Ill-Posed Problems, Longman, Essex, 1995.

[9] M. Hanke and P. C. Hansen, Regularization methods for large-scale problems, Surv. Math. Ind., 3 (1993), pp. 253-315.

[10] P. C. Hansen, Regularization tools: A Matlab package for analysis and solution of discrete ill-posed problems, Numer. Algorithms, 6 (1994), pp. 1-35.

[11] P. C. Hansen, Rank-Deficient and Discrete Ill-Posed Problems, SIAM, Philadelphia, 1998.

[12] M. Hochbruck and C. Lubich, Error analysis of Krylov methods in a nutshell, SIAM J. Sci. Comput., 19 (1998), pp. 695-701.

[13] V. A. Morozov, On the solution of functional equations by the method of regularization, Soviet Math. Dokl., 7 (1966), pp. 414-417.

[14] R. Plato, On the discrepancy principle for iterative and parametric methods to solve linear ill-posed equations, Numer. Math., 75 (1996), pp. 99-120.

15] Y. Saad, Iterative Methods for Sparse Linear Systems, PWS, Boston, MA, 1996.

[16] Y. Saad and M. H. Schultz, GMRES: a generalized minimal residual method for solving nonsymmetric linear systems, SIAM J. Sci. Stat. Comput., 7 (1986), pp. 856-869.

[17] P. Å. Wedin, Perturbation theory for pseudo-inverses, BIT, 13 (1973), pp. 217-232. 


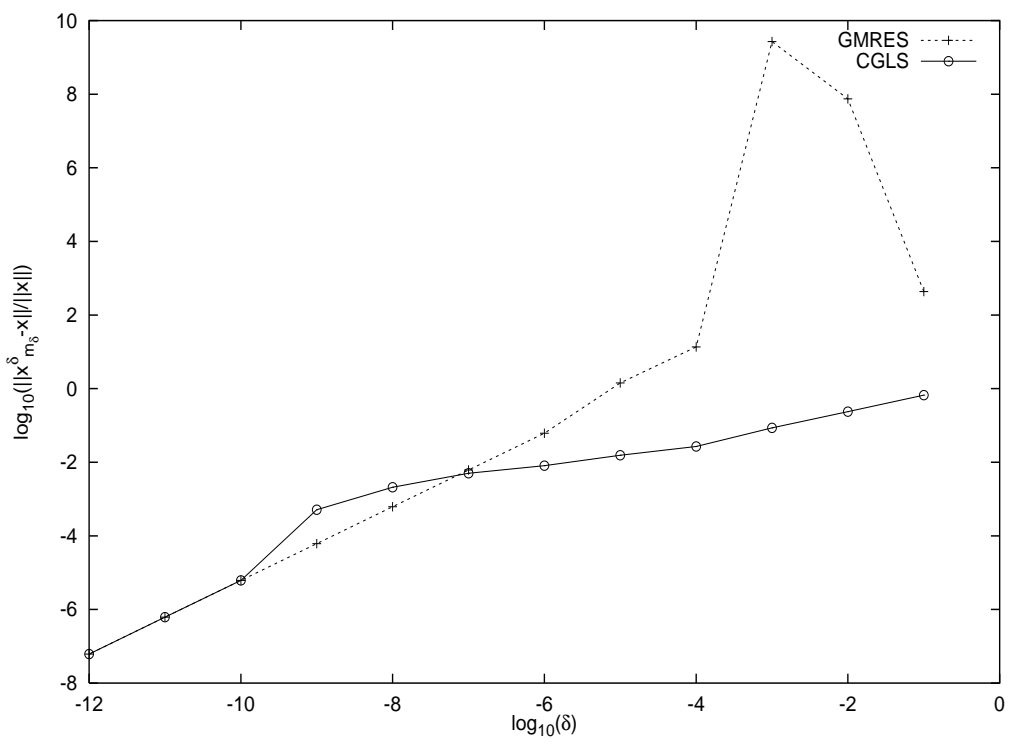

FIG. 4.1. Example 4.1: Relative error norms of the iterates $x_{m_{\delta}}^{\delta}$ computed by the GMRES and CGLS methods.

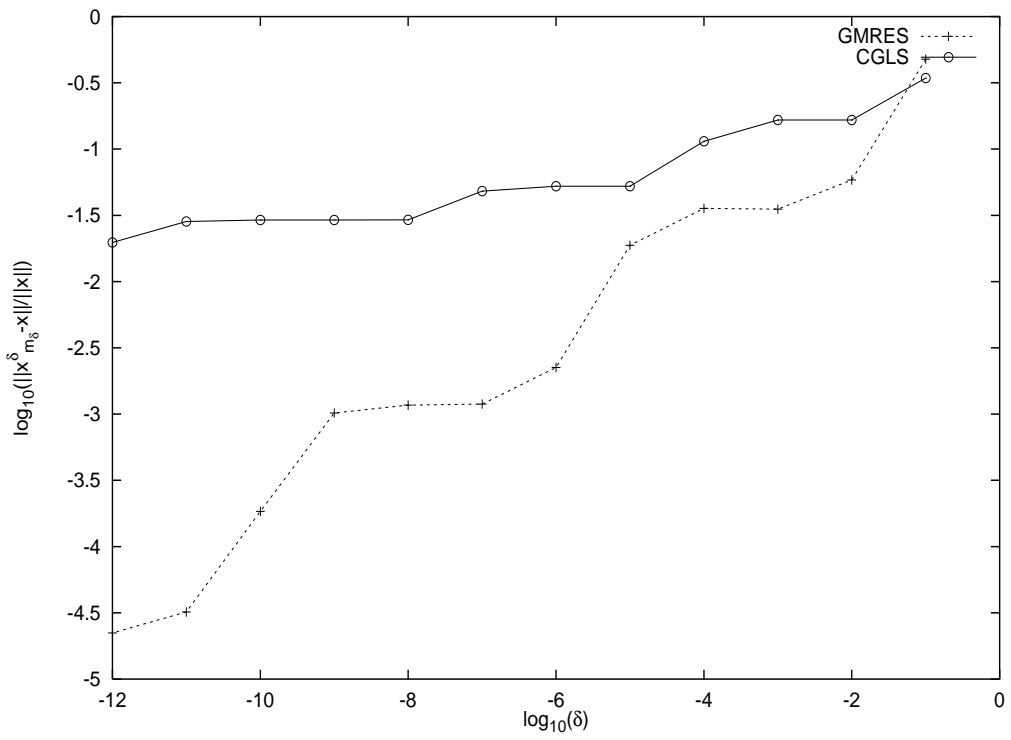

FIG. 4.2. Example 4.2: Relative error norms of the iterates $x_{m_{\delta}}^{\delta}$ computed by the GMRES and CGLS methods. 